\title{
Strong enhancement of magnetic order from bulk to stretched monolayer FeSe as Hund's metals
}

\author{
Chang-Youn Moon $\mathbb{i D}^{1 凶}$
}

Despite of the importance of magnetism in possible relation to other key properties in iron-based superconductors, its understanding is still far from complete especially for FeSe systems. On one hand, the origin of the absence of magnetic orders in bulk FeSe is yet to be clarified. On the other hand, it is still not clear how close monolayer FeSe on $\mathrm{SrTiO}_{3}$, with the highest transition temperature among iron-based superconductors, is to a magnetic instability. Here we investigate magnetic properties of bulk and monolayer FeSe using dynamical mean-field theory combined with density-functional theory. We find that suppressed magnetic order in bulk FeSe is associated with the reduction of interorbital charge fluctuations, an effect of Hund's coupling, enhanced by a larger crystal-field splitting. Meanwhile, spatial isolation of Fe atoms in expanded monolayer FeSe leads into a strong magnetic order, which is completely destroyed by a small electron doping. Our work provides a comprehensive understanding of the magnetic order in iron-based superconductors and other general multi-orbital correlated systems as Hund's metals.

npj Computational Materials (2020)6:147; https://doi.org/10.1038/s41524-020-00414-3

\section{INTRODUCTION}

Magnetism is one of universal features found in iron-based superconductors (IBS) as superconductivity generally appears in the vicinity of antiferromagnetic (AFM) phase with a specific stripe-type ordering pattern, from which electron pairing mechanisms of the magnetic origin were introduced ${ }^{1-7}$. Furthermore, nematicity (spontaneous breaking of four-fold rotational symmetry of tetragonal phase), magnetism, and superconductivity in IBS are thought to be closely related ${ }^{8-11}$. In this context, understanding magnetism can be a starting point to unravel the complex interdependence of these properties. In terms of magnetism, FeSe holds a unique position among general IBS as bulk FeSe has no magnetic ordered phase unlike most of other materials ${ }^{12-14}$, whose underlying mechanism is still not well understood. FeSe is also of great interest due to the highest superconducting transition temperature among IBS when its monolayer (ML) is on $\mathrm{SrTiO}_{3}$ substrate ${ }^{15-18}$. Whether or not ML $\mathrm{FeSe} / \mathrm{SrTiO}_{3}$ is close to a magnetic instability is therefore an intriguing question.

Meanwhile, there is a general consensus that the electron correlation should be taken into account to properly understand material properties of this system ${ }^{19-22}$. Since it is a multi-orbital system in which all five $d$ orbital bands are crossing or near the Fermi energy $\left(E_{F}\right)$, Hund's coupling $J_{H}$ is an indispensable part of interactions as well as the intraorbital Coulomb repulsion $U$, and IBS in the correlated metallic state are often described as Hund's metals ${ }^{20,23-25}$. In this material state, reduced interorbital Coulomb repulsion $U^{\prime}=U-2 J_{H}$ and the tendency to promote parallel spin alignment cooperatively decouple the five $d$ orbital components, which is signaled by the suppression of interorbital charge fluctuations. Consequently, coherent and incoherent states can coexist and some orbitals are close to Mott transition while the others are still itinerant. Since this orbital selectivity is known to be enhanced in $\mathrm{FeSe}^{21-23,26,27}$, its magnetic properties would be better understood in the context of Hund's metal physics.

In this work, a systematic comparative study on the magnetic properties of FeSe in different forms and a reference IBS, LaFeAsO, is performed using a density-functional theory plus dynamical mean-field theory (DFT + DMFT). It is found that the interorbital charge fluctuations are greatly reduced between $e_{g}$ and $t_{2 g}$ orbitals for bulk FeSe due to its large crystal-field splitting and the resultant strong orbital decoupling induced by the Hund's coupling. Consequently the total charge fluctuation are enhanced leading to a largely reduced ordered magnetic moment compared with $\mathrm{LaFeAsO}$, consistently with the absence of magnetic order in bulk FeSe in experiments. In contrast, increased fluctuating magnetic moment and suppressed total charge fluctuation due to the increased interatomic distance and the reduced dimentionality result in a large ordered magnetic moment in expanded ML FeSe with the lattice constant of that on $\mathrm{SrTiO}_{3}$. Thus, the stark contrast of the magnetic order between bulk and ML FeSe is explained in terms of Hund' metal properties within an unified framework. Small electron doping is found to effectively destroy the magnetic order in this system, implying that the superconductivity in $\mathrm{ML} \mathrm{FeSe} / \mathrm{SrTiO}_{3}$ is in the vicinity of magnetic order.

\section{RESULTS}

Magnetic susceptibility

Three different materials are considered in this work; namely, LaFeAsO as an archetypal IBS, bulk FeSe, and freestanding ML FeSe tensile-strained to the lattice constant of $\mathrm{ML} \mathrm{FeSe} / \mathrm{SrTiO}_{3}$, $3.90 \AA^{17}$. A recent DFT + DMFT study demonstrated that the main effect of defect-free $\mathrm{SrTiO}_{3}$ substrate on the electronic structure of $\mathrm{ML} \mathrm{FeSe}$ is to increase the Se-Fe-Se angle through increasing the lattice constant of ML FeSe ${ }^{28}$, and an earlier DFT study suggested a similar conclusion ${ }^{29}$. Therefore, strained freestanding ML FeSe is expected to capture most of the essential features of magnetic properties of that on $\mathrm{SrTiO}_{3}$ as well. Electron doping, another important possible substrate effect, will be also discussed in the later part of this work.

Figure 1 displays the imaginary part of the magnetic susceptibility, $X_{m}^{\prime \prime}$, as a function of momentum and frequency, for the three materials in the paramagnetic (PM) phase. The magnetic

\footnotetext{
${ }^{1}$ Advanced Instrumentation Institute, Korea Research Institute of Standards and Science, Yuseong, Daejeon, Republic of Korea. ${ }^{凶}$ email: cymoon@kriss.re.kr
} 
susceptibility is estimated within DFT + DMFT method from the Bethe-Salpeter equation, using fully momentum and frequency dependent interacting DFT + DMFT one-particle lattice Green's function and local two-particle vertex function obtained from the DMFT impurity solver $^{30} . X_{m}^{\prime \prime}$ for LaFeAsO exhibits a typical spin excitation spectrum for IBS, with largest weights at $q=(1,0)$ near zero frequency indicating the magnetic instability for the stripetype AFM order and also with high energy excitations near $q=(1$, $1)$, as can be seen in previous similar calculations ${ }^{6,30-32}$. Meanwhile, low-energy spin fluctuations are much suppressed for bulk FeSe indicating the weakened tendency for the magnetic order in accordance with its absence in experiments. Considerable amount of spectral weights near zero energy are relocated to near $100 \mathrm{meV}$, implying that some higher frequency processes are involved in the magnetic order suppression. Finally, ML FeSe exhibits the overall increase of spectral weights as well as the recovered dominance of low-energy fluctuations over high energy ones compared with bulk FeSe, indicating a much stronger tendency for the magnetic order. In this case, however, strongest low-energy excitations are not at $q=(1,0)$, but slightly shifted from it toward $q=(1,1)$ suggesting an incommensurate magnetic order. Besides the spin fluctuation, as the orbital degree of freedom is considered another candidate to drive the nematic

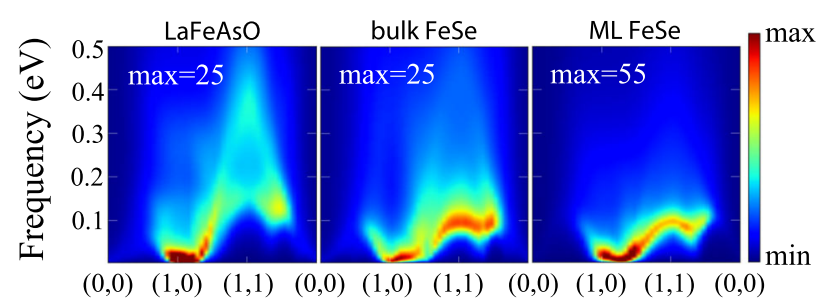

$(H, K)$ (r. 1. u.)

Fig. 1 Imaginary part of magnetic susceptibility. For LaFeAsO, bulk FeSe, and ML FeSe, respectively. $x$-axis is for the momentum transfer $\mathbf{q}=(H, K, L=1)$ in the reciprocal lattice unit (r. I. u.) of one-Fe-unitcell, and $y$-axis is for the frequency. order and/or the superconductivity in these materials, we also estimate the orbital susceptibility (see Supplementary Fig. 1 and Note 1). Only very weak low-energy excitations are found for all the three materials, indicating that DFT + DMFT method does not support the existence of orbital orders in these materials.

\section{Trends in local quantities}

We perform a systematic analysis for the trend of local correlations to understand the properties found in the susceptibility results. Ordered magnetic moment $\left\langle S_{z}\right\rangle$ on a Fe atom is estimated in the stripe-type AFM phase and found to vary from 0.70 to 0.43 and $1.00 \mu_{B}$ for LaFeAsO, bulk and ML FeSe, respectively. We can see that the magnetic order is suppressed and then greatly enhanced for bulk and ML FeSe compared with $\mathrm{LaFeAsO}$ as predicted by magnetic susceptibility results in the PM phase in Fig. 1, and also in qualitative agreement with the experimental observation of no magnetic order for bulk FeSe. Usually magnetic order is strong in materials with strong electron correlation, and greatly reduced ordered moment of bulk FeSe is rather puzzling since it is considered to be more correlated than $\mathrm{LaFeAsO}$. Indeed, mass enhancement factor, $1 / Z=1-\left.\frac{\partial \Sigma(\omega)}{\partial \omega}\right|_{\omega=0}$, is found to increases considerably for $t_{2 g}$ orbitals, especially $d_{x y}$ as shown in Fig. 2a. Although $e_{g}$ orbitals become less correlated from LaFeAsO to bulk FeSe, the fluctuating magnetic moment $\left(\left\langle S^{2}\right\rangle^{1 / 2}\right)$, which reflects the overall correlation strength, slightly increases in Fig. $2 b$ suggesting that the suppressed magnetic order in bulk FeSe cannot be understood by the overall correlation strength of the material. Meanwhile, mass enhancement increases for all the orbitals for ML FeSe in Fig. 2a along with the fluctuating moment in Fig. $2 b$ defining this material most correlated among the three.

Using the same $U^{\prime} s$ and $J$ 's for all the materials in the present study (see Supplementary Note 2), the variation of correlation strength can be attributed mainly to that of the interatomic distance and orbital occupations. In spite of the large reduction of the Fe-anion distance from 2.42 to $2.39 \AA$ for LaFeAsO and bulk FeSe, respectively, $t_{2 g}$ orbitals become much more correlated while $e_{g}$ orbitals exhibit the opposite behavior to produce a large orbital differentiation in bulk FeSe. As can be seen in Fig. 2c, it
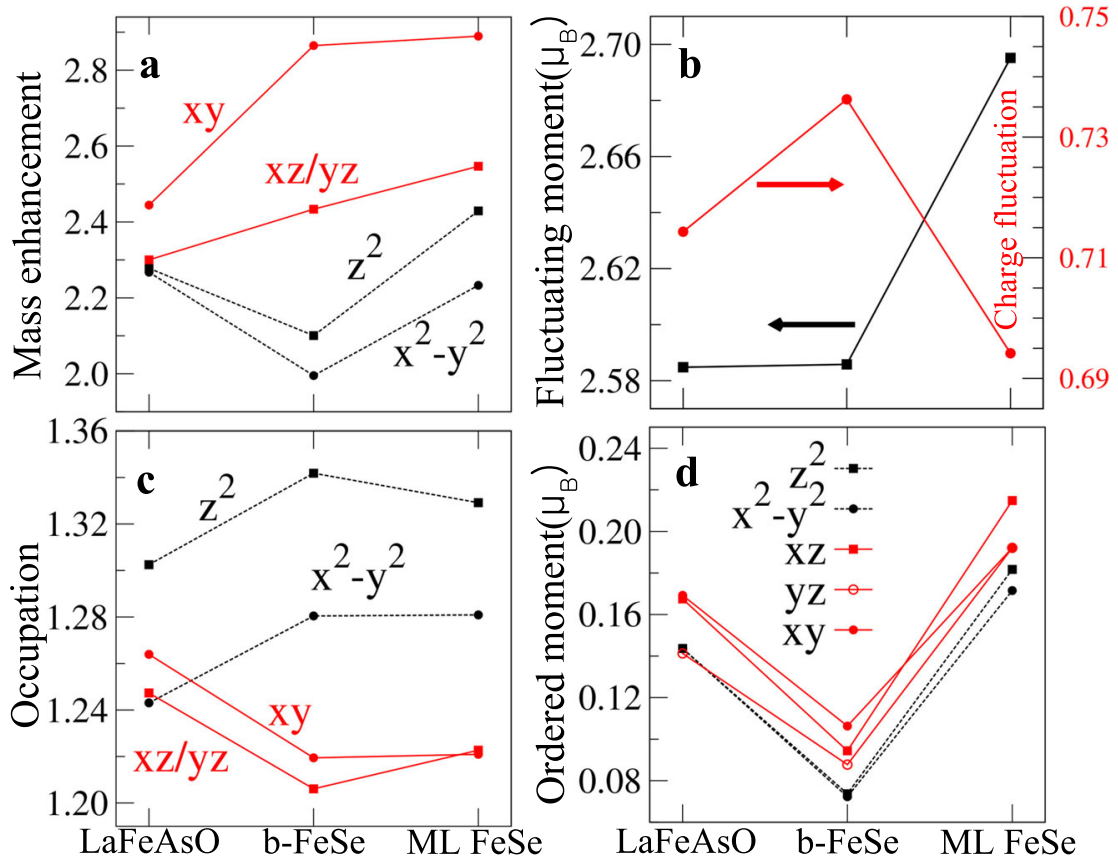

Fig. 2 Local quantities. a Orbital-resolved mass enhancement, b fluctuating moment and local charge fluctuation, c orbital occupations, and d orbital-resolved ordered moments on an iron atom for LaFeAsO, bulk FeSe (b-FeSe), and ML FeSe. Ordered moment in d are estimated in the AFM phase, while others in the PM phase. $U=5 \mathrm{eV}$ and $J=0.8 \mathrm{eV}$ are used. 
results from the large difference of occupation numbers between $t_{2 g}$ and $e_{g}$ orbitals, which are essentially decoupled in a Hund's metal $^{20,24,25}$, indicating a large crystal-field splitting in bulk FeSe. We estimate that all five Fe- $d$ orbital levels lie within the range of $0.25 \mathrm{eV}$ for $\mathrm{LaFeAsO}$ while the range increases to $0.48 \mathrm{eV}$ for bulk FeSe indeed confirming the enhanced crystal-field splitting in bulk FeSe. Noteworthy is that even in bulk FeSe the crystal-field splitting is smaller than $J$ value adopted in this work, $0.8 \mathrm{eV}$, so that the Hund's coupling still plays a major role in the local correlation overall five $d$ orbitals in this material. The overall increase of mass enhancement of ML FeSe can then be related to the elongation of Fe-anion bond to $2.40 \AA$ due to the applied strain, considering that its orbital occupations do not change much from those of bulk FeSe. Also, the kinetic energy reduction in a two-dimensional system is expected to further contribute to the stronger overall correlation in ML FeSe, especially for $d_{x z / y z}$ and $d_{z^{2}}$ orbitals.

In a Hund's metal, the local charge fluctuation $\left\langle n^{2}\right\rangle-\langle n\rangle^{2}$ where $n$ is the local density operator on an atom, which quantifies the charge delocalization, can be sizable even in the strongly correlated case because the electron correlation comes from the dominance of high-spin states in the local subspace while electrons can hop through unoccupied orbitals ${ }^{24}$. Hund's coupling promotes a fluctuating moment while this active charge fluctuation hinders its static order leading to the much reduced magnitude of ordered moment compared with the fluctuating moment ${ }^{20,23,31}$. Figure $2 \mathrm{~b}$ indeed shows the correlation between the charge fluctuation and ordered moment, where the enhanced charge fluctuation for bulk FeSe accounts for its suppressed ordered moment of $0.43 \mu_{B}$ compared with $0.70 \mu_{B}$ of LaFeAsO while the suppressed charge fluctuation coincides with the enhanced ordered moment of $1.00 \mu_{B}$ for ML FeSe.

To understand the variation of charge fluctuation over materials, orbital-resolved charge fluctuations defined as,

$\left\langle n_{a} n_{\beta}\right\rangle-\left\langle n_{a}\right\rangle\left\langle n_{\beta}\right\rangle$

where $a$ and $\beta$ are orbital indexes, are estimated and listed in Table 1. Diagonal elements represent intraorbital charge fluctuations and are roughly correlated with respective orbital occupations where orbitals close to the half-integer filling 1.5 have higher charge fluctuations. Meanwhile, off-diagonal elements correspond to interorbital charge fluctuations and have negative values, due to the interorbital Coulomb repulsion $U^{\prime}$. Their small (absolute) values are the signature of the orbital decoupling which characterizes Hund's metals, and can contribute to increase the total charge fluctuation of an atom. Larger overlap between $e_{g}$ and $t_{2 g}$ orbitals enhances $U^{\prime}$, and hence interorbital charge fluctuations are dominant between them. From $\mathrm{LaFeAsO}$ to bulk FeSe, intraorbital charge fluctuation slightly increases or remain almost the same for $e_{g}$ orbitals, while it is considerably suppressed for $t_{2 g}$ orbitals (by $18 \%$ for $d_{x y}$ ) so that there are large differences between $e_{g}$ and $t_{2 g}$ orbitals, following the trend of orbital occupations shown in Fig. 2c. In contrast, interorbital components greatly increase (decreased absolute values), especially between $d_{x^{2}-y^{2}}$ and $d_{x y}$ orbitals by over $50 \%$, which overcomes the overall reduction of intraorbital components and produce the net increase of total charge fluctuation as displayed in Fig. 2b. Hund's coupling keeps the the magnitude of the local spin on an iron atom (i.e., $S^{2}$ ) finite in both materials as indicated by their similar fluctuating moments in Fig. $2 \mathrm{~b}$ and orbital-resolved spin fluctuations (see Supplementary Table 1 and Note 3). Meanwhile, the charge fluctuation enhances the chance of spin flip processes of this local spin as a whole, not losing Hund's coupling energy, to result in the contrasting ordered moments between LaFeAsO and bulk FeSe as shown in Fig. $2 \mathrm{~d}$ where every orbital component of the ordered moment is reduced for the latter compared with the former. As mentioned earlier, the enhanced spin flip processes reducing the ordered moment in bulk FeSe can be associated with the 100 meV spin excitations in Fig. 1.
Table 1. Orbital-resolved charge fluctuations in the PM phase.

\begin{tabular}{lllll}
\hline & $z^{2}$ & $x^{2}-y^{2}$ & $x z / y z$ & $x y$ \\
& LaFeAsO & & & \\
\hline$z^{2}$ & 0.229 & 0 & -0.037 & -0.003 \\
$x^{2}-y^{2}$ & 0 & 0.212 & -0.013 & -0.046 \\
$x z / y z$ & -0.037 & -0.013 & $0.197(0.012)$ & -0.015 \\
$x y$ & -0.003 & -0.046 & -0.015 & 0.222 \\
& & & & \\
\hline & bulk FeSe & & -0.032 & -0.004 \\
\hline$z^{2}$ & 0.238 & 0.001 & -0.014 & -0.021 \\
$x^{2}-y^{2}$ & 0.001 & 0.210 & $0.184(0.013)$ & -0.014 \\
$x z / y z$ & -0.032 & -0.014 & -0.014 & 0.182 \\
$x y$ & -0.004 & -0.021 & & \\
\hline & & & & -0.005 \\
\hline$z^{2}$ & Monolayer FeSe & -0.002 & -0.035 & -0.023 \\
$x^{2}-y^{2}$ & -0.002 & 0.208 & -0.016 & 0.015 \\
$x z / y z$ & -0.035 & -0.016 & $0.188(0.009)$ & 0.182 \\
$x y$ & -0.005 & -0.022 & -0.015 & \\
\hline
\end{tabular}

The definition is shown as Eq. (1) in the main text. Diagonal elements are intraorbital charge fluctuations, which quantify how much the electron in the orbital is itinerant, while off-diagonal ones are interorbital charge fluctuations, which are negative because of the Coulomb repulsion among orbitals. A number in a parenthesis represents the interorbital element between $d_{x z}$ and $d_{y z}$ orbitals, and the number in front of it is the intraorbital element of $d_{x z}$ and $d_{y z}$, which are the same. Interorbital components between $d_{x^{2}-y^{2}}$ and $d_{x y}$ are shown in bold font to emphasize their stark contrast between LaFeAsO and bulk FeSe. $U=5 \mathrm{eV}$ and $J=0.8 \mathrm{eV}$ are used.

The pronounced suppression of interorbital charge fluctuations in bulk FeSe can be attributed to the large difference of its intraorbital components between $e_{g}$ and $t_{2 g}$ orbitals shown in Table 1, as the interorbital fluctuation is expected to be suppressed between orbitals which fluctuate incoherently to each other with very different rates. Since the difference in intraorbital charge fluctuations among orbitals can be mainly accounted for by that in orbital occupations as mentioned above, their larger difference in bulk FeSe is the direct consequence of the larger crystal-field splitting. In short, the suppressed magnetic order in bulk FeSe compared with $\mathrm{LaFeAsO}$ is a result of its relatively large crystal-field splitting which produces a strong orbital selectivity by the action of Hund's coupling (see Supplementary Note 4). Meanwhile, orbital occupations do not change much from bulk to $\mathrm{ML} \mathrm{FeSe}$ in Fig. 2c and hence neither do interorbital charge fluctuations and other components in Table 1. The decreased total charge fluctuation of $\mathrm{ML}$ FeSe is a cooperative result from all of the components with small and even contributions, without a single dominant one. Together with the increased fluctuating moment as shown in Fig. 2b, the suppressed charge fluctuation leads to a strong magnetic order of $1 \mu_{B}$ and can be considered as a natural consequence of localized orbitals with increased interatomic distances compared with bulk FeSe.

\section{Effects of doping on ML FeSe}

The stabilization of the AFM phase in ML FeSe on $\mathrm{SrTiO}_{3}$ has been also predicted by previous DFT calculations ${ }^{29,33,34}$, however, with large ordered moments of over $2 \mu_{B}$, which are likely overestimated as is a well-known general property of DFT on IBS. A recent experimental work indeed confirmed an AFM order in this 

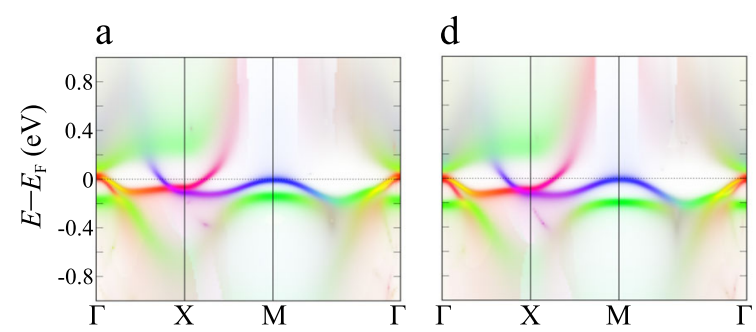

b

e
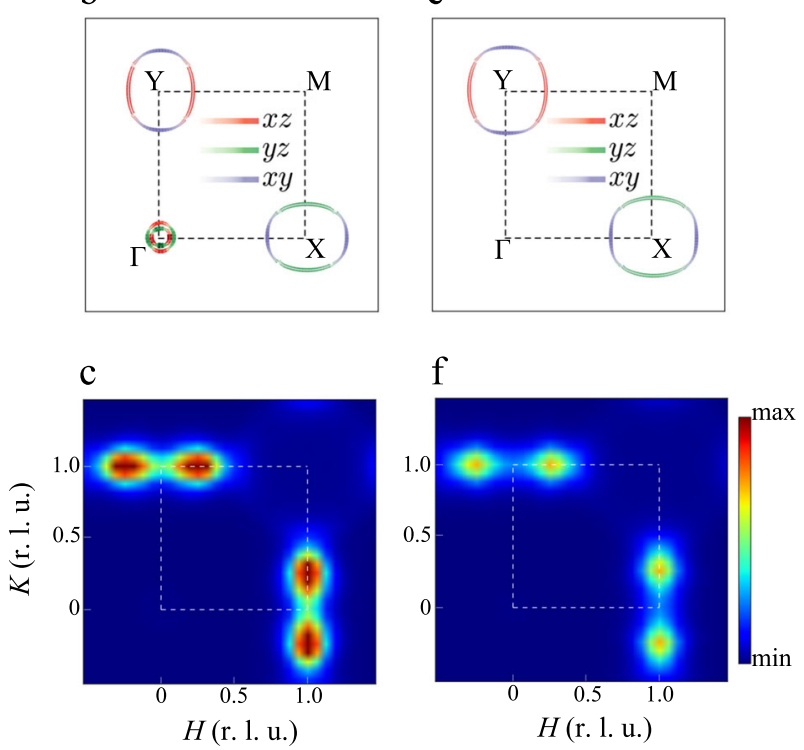

Fig. 3 Effect of doping on band structure and spin susceptibility of ML FeSe. a Orbital-resolved spectral functions $A(\mathbf{k}, \omega)$ along the high-symmetry points in the one-Fe-unitcell for the ML FeSe. Red and blue represent $d_{x z / y z}$ and $d_{x y}$ components, respectively, while green is for $e_{g}$ orbitals. b FS in the two-dimensional BZ of one-Feunitcell for ML FeSe, evaluated by the real part of the complex energy eigenvalues from the DFT + DMFT calculation. Weight of an orbital component is represented by the depth of a color as well as the thickness of the line. $\mathbf{c}, X_{m}^{\prime \prime}(\mathbf{q}, \omega=5 \mathrm{meV})$ in the same BZ as that in $\mathbf{b}$. $\mathbf{d}-\mathbf{f}$ are counterparts of $\mathbf{a}-\mathbf{c}$ for the $0.12 e^{-} / \mathrm{Fe}$ doped ML FeSe, respectively.

system using magnetic exchange bias effect measurement ${ }^{35}$, though neither the ordering vector nor the ordered moment could be determined. It is also found that the magnetic order disappears for the electron doped sample where superconductivity can arise. To investigate the effect of doping on the electronic and magnetic properties of ML FeSe, $0.12 e^{-} / \mathrm{Fe}$ is added as determined on the superconducting sample by an earlier experimental study ${ }^{17}$. Figure $3 a$ and $d$ shows the spectral function $\mathrm{A}(k, \omega)$ with orbital characters in the BZ of one-Fe-atom unitcell for undoped and electron doped ML FeSe systems, respectively. Two hole bands around $\Gamma$ and electron bands around $X$ are mainly of $d_{x z / y z}$ character, while another hole band at $M$ is from $d_{x y}$ orbital. Meanwhile, $e_{g}$ orbital components are located relatively farther from the $E_{F}$. Although some spectral weights are above the $E_{F}$ for the hole band at $M$ due to its incoherence, its real eigenvalues, which determine the peak positions of $A(k, \omega)$ are actually below the $E_{F}$, so in the FS plot in Fig. $3 \mathrm{~b}$ and e, no hole FS is shown around $M$. Around $\Gamma$, on the other hand, two small hole FS exist for undoped $\mathrm{ML}$ FeSe, while they sink below the $E_{F}$ for the doped case. Consequently, no hole surface is present for the doped ML $\mathrm{FeSe}$, in agreement with experimental observations on ML FeSe/ $\mathrm{SrTiO}_{3}$ system ${ }^{16,17,22,36}$ as well as previous DFT+DMFT calculations ${ }^{37,38}$. In Fig. $3 c$ and $f, X_{m}^{\prime \prime}(q, \omega=5 \mathrm{meV})$ in the PM phase is displayed to figure out how the static magnetic order evolves with doping. In the undoped case, static order is predicted slightly off the stripe-type AFM ordering vector as is already seen in Fig. 1. Despite of significant renormalization of the noninteracting susceptibility $x_{0}$ by the local two-particle vertex to form the fully interacting $x^{31}$, the FS nesting which features the structure of $X_{0}$ still plays a non-negligible role in stabilizing magnetic ordering ${ }^{23}$. Indeed, one can see that the nesting vectors connecting the hole FSs at $\Gamma$ and the electron FS at $X$ or $Y$ with same orbital characters in Fig. $3 b$ roughly coincides with the peak positions of $X_{m}^{\prime \prime}$ in Fig. 3c. Even though the hole FS is absent in Fig. 3e by the electron doping, actually the hole bands are just below $E_{F}$ as shown in Fig. $3 \mathrm{~d}$ so that the overall nesting condition is not very different from the undoped case. Consequently, the peak position in $X_{m}^{\prime \prime}$ plot in Fig. $3 f$ is almost the same as in Fig. 3c, with only the overall excitation magnitude greatly reduced. The suppressed low-energy excitation and tendency for a magnetic order rather result from the local two-particle vertex, which includes effects of overall increase of local orbital occupations away from the integer filling by doping, which should suppress the fluctuating moment and enhance charge fluctuations. Zero ordered moment is obtained in the stripe-type AFM calculation for $0.12 e^{-} /$Fe doped ML FeSe, in consistence with our $X_{m}^{\prime \prime}$ result in the PM phase and also with the suppressed magnetic order by electron doping found experimentally ${ }^{35}$ as mentioned above. This large sensitivity of magnetic order on doping therefore results from local correlations, which are well described within the DFT + DMFT method.

\section{DISCUSSION}

Our result, that strong magnetic order in strained ML FeSe is destroyed by electron doping on the level where superconductivity is known to appear, implies the close proximity of magnetism to the superconductivity in $\mathrm{ML} \mathrm{FeSe} / \mathrm{SrTiO}_{3}$, imposing a definite constraint on the electron pairing mechanism in this system. Among various pairing scenarios taking into account the absence of hole FS around $\Gamma$, our results are most consistent with the "bootstrap" mechanism where electron FSs and "incipient" band (hole band below $E_{F}$ ) have opposite sign gaps $\left(s_{ \pm}\right)^{39-41}$. This mechanism requires cooperative interplay of attractive $q \sim(0,0)$ interaction (e.g., by phonon) and repulsive $q \sim(1,0)$ interaction whose existence is identified in our study as the incommensurate spin excitation. Meanwhile, $q \sim(1,1)$ interaction connecting separate electron FSs, as required by other scenarios such as "nodeless $d^{\prime \prime 24-44}$, "sign-preserving s"45,46, and "bonding-antibonding $s^{\prime \prime 47,48}$, is identified from neither spin nor orbital excitations as shown in Fig. 1 and Supplementary Fig. 1, respectively, although nonlocal correlations not included in the DFT + DMFT scheme might help stabilize low-energy orbital fluctuations ${ }^{49}$.

Our work casts new light on understanding the dramatic variation of ordered moment in IBS, including the long standing puzzle of the absence of magnetic order in bulk FeSe. Besides the overall correlation strength as reflected on the size of fluctuating moment, orbital-specific correlations are also important in determining the magnetic order, as large difference in intraorbital charge fluctuation among orbitals, e.g., induced by enhanced crystal-field splitting in case of bulk FeSe, can give rise to suppressed interorbital charge fluctuation and eventually result in reduced ordered moment of each orbital. As our calculated ordered moment of $0.4 \mu_{B}$ for bulk FeSe is still non-zero but certainly smaller than for other materials considered, even tiny amount of excess electrons generated by intrinsic small excess Fe or Se deficiency ${ }^{13}$ might easily lead to completely destroyed magnetic order. We expect that other materials which deviate from the general trend of ordered moment according to the correlation strength and fluctuating moment as shown in Fig. 1 in 
ref. ${ }^{23}$, such as LiFeAs which also exhibits no magnetic phase, can possibly be understood with a similar mechanism.

In summary, magnetic properties of bulk and tensile-strained ML FeSe are investigated using DFT + DMFT method. Magnetic susceptibility in the PM state indicates suppressed and strongly enhanced magnetic orders at and near the stripe-type AFM ordering vector for bulk and ML FeSe, respectively. Bulk FeSe is found to have a pronounced orbital decoupling, i.e., strongly reduced interorbital charge fluctuations between $e_{g}$ and $t_{2 g}$ orbitals, which result from its large crystal-field splitting and are manifested by the Hundness of general IBS materials. We suggest that the consequently enhanced total charge fluctuation suppresses the static ordering of the fluctuating local spin formed by Hund's coupling. On the other hand, magnetic order is strongly enhanced in ML FeSe due to enlarged fluctuating moment and slower charge fluctuations caused by more isolated Fe atoms with the larger lattice constant of the material. We find that the magnetic order disappears after $0.12 e^{-}$doping in ML FeSe along with the hole FSs in the BZ, suggesting a possible relationship between the magnetic order and the superconductivity in $\mathrm{ML}$ $\mathrm{FeSe} / \mathrm{SrTiO}_{3}$

\section{METHODS}

\section{Details of DFT + DMFT calculation}

We use the modern implementation of DFT + DMFT method within allelectron embedded DMFT approach ${ }^{50}$, where in addition to correlated Fe atoms the itinerant states of Se are included in the Dyson self-consistent equation. The strong correlations on the $\mathrm{Fe}$ ion are treated by DMFT, adding self-energy $\Sigma(\omega)$ on a quasi atomic orbital in real space, to ensure stationarity of the DFT + DMFT approach. The self-energy $\Sigma(\omega)$ contains all Feynman diagrams local to the $\mathrm{Fe}$ ion. No downfolding or other approximations were used, and the calculations are all-electron as implemented in ref. ${ }^{48}$, which is based on Wien $2 k^{51}$. We employ LDA exchange-correlation functional ${ }^{52,53}$, and the quantum impurity model was solved by the continuous time quantum Monte Carlo (CTQMC) impurity solver $^{54}$. Fixed $U=5.0 \mathrm{eV}$ and $J=0.8 \mathrm{eV}$ values are used for all the three materials (see Supplementary Note 2) as in the previous work studying a number of different IBS using the same methodological scheme with the one adopted in this study ${ }^{23}$. These values are also in reasonable agreement with those employed in a previous LDA $+U$ study for another ferrous material $\left.\right|^{55}$. We use the Slater parametrization of the Coulomb interaction in this study, and our $U$ and $J$ parameters are defined with respect to the three Slater parameters in such a way that $F^{0}=U, F^{2}=112 / 13 \mathrm{~J}$, and $F^{4}=$ $70 / 13 \mathrm{~J}$. Thus this is not to be mistaken for being equivalent to use a single $J$ value averaged over different orbitals within more commonly used Kanamori parametrization, and the anisotropy of interactions among different orbitals is taken into account in our calculation with the spherical symmetry assumed. For detailed information on the Coulomb parametrization employed in this work, refer to http://hauleweb.rutgers.edu/ tutorials/CoulombUexplain.html. Further useful discussions on the treatment of Coulomb interaction also can be found in Correlated Electrons: From Models to Materials, ISBN 978-3-89336-796-2. BZ integration is done on the $14 \times 14 \times 9 \mathrm{k}$-point mesh for the 2-Fe atom unitcell of bulk FeSe, and equivalent or similar meshes on other structures. Calculations for PM phases are done at $T=387 \mathrm{~K}$, and magnetic phases are obtained at $T=$ $116 \mathrm{~K}$. All atomic positions are fully optimized with lattice constants fixed to experimental values ${ }^{13,56}$ within DFT + DMFT scheme by minimizing forces obtained from the derivative of stationary-free energy functional as implemented in ref. ${ }^{57}$, where it is shown how the inclusion of spin fluctuation in DFT + DMFT naturally leads to significantly better agreement of Se position with experimental values than DFT only calculations. Optimized atomic positions of As and $\mathrm{Se}$ in the internal lattice unit are 0.1537 and 0.2670 with respect to the Fe plane for LaFeAsO and bulk FeSe, which show good agreements with the experimental values of $0.1517^{56}$ and $0.2672^{13}$

\section{DATA AVAILABILITY}

The data that support the findings of this study are available from the corresponding author upon reasonable request.
Received: 21 April 2020; Accepted: 7 September 2020;

Published online: 01 October 2020

\section{REFERENCES}

1. Mazin, I. I., Singh, D. J., Johannes, M. D. \& Du, M. H. Unconventional superconductivity with a sign reversal in the order parameter of $\mathrm{LaFeAsO}_{1-x} \mathrm{~F}_{x}$. Phys. Rev. Lett. 101, 057003 (2008).

2. Zhang, J., Sknepnek, R., Fernandes, R. M. \& Schmalian, J. Orbital coupling and superconductivity in the iron pnictides. Phys. Rev. B 79, 220502(R) (2009).

3. Lee, C. H. et al. Incommensurate spin fluctuations in hole-overdoped superconductor $\mathrm{KFe}_{2} \mathrm{As}_{2}$. Phys. Rev. Lett. 106, 067003 (2011).

4. Ji, G. F. et al. Simultaneous optimization of spin fluctuations and superconductivity under pressure in an iron-based superconductor. Phys. Rev. Lett. 111, 107004 (2013)

5. Dai, Y. M. et al. Hidden T-linear scattering rate in $\mathrm{Ba}_{0.6} \mathrm{~K}_{0.4} \mathrm{Fe}_{2} \mathrm{As}_{2}$ revealed by optical spectroscopy. Phys. Rev. Lett. 111, 117001 (2013).

6. Yin, Z. P., Haule, K. \& Kotliar, G. Spin dynamics and orbital-antiphase pairing symmetry in iron-based superconductors. Nat. Phys. 10, 845-850 (2014).

7. Allan, M. P. et al. Identifying the 'fingerprint' of antiferromagnetic spin fluctuations in iron pnictide superconductors. Nat. Phys. 11, 177-182 (2015).

8. Fernandes, R. M., Chubukov, A. V. \& Schmalian, J. What drives nematic order in iron-based superconductors? Nat. Phys. 10, 97-104 (2014).

9. Yamakawa, Y., Onari, S. \& Kontani, H. Nematicity and magnetism in FeSe and other families of Fe-based superconductors. Phys. Rev. X 6, 021032 (2016).

10. Matsuura, $\mathrm{K}$. et al. Maximizing $\mathrm{T}_{c}$ by tuning nematicity and magnetism in $\mathrm{FeSe}_{1-x}$ $\mathrm{S}_{x}$ superconductors. Nat. Commun. 8, 1143 (2017).

11. Li, J. et al. Nematic superconducting state in iron pnictide superconductors. Nat. Commun. 8, 1880 (2017).

12. Hsu, F. C. et al. Superconductivity in the PbO-type structure $a$-FeSe. Proc. Natl. Acad. Sci. USA 105, 14262-14264 (2008).

13. McQueen, T. M. et al. Extreme sensitivity of superconductivity to stoichiometry in $\mathrm{Fe}_{1 . \delta}$ Se. Phys. Rev. B 79, 014522 (2009).

14. Baek, S.-H. et al. Orbital-driven nematicity in FeSe. Nat. Mater. 14, 210-214 (2015).

15. Wang, Q.-Y. et al. Interface-induced high-temperature superconductivity in single unit-cell FeSe films on $\mathrm{SrTiO}_{3}$. Chin. Phys. Lett. 29, 037402 (2012).

16. $\mathrm{He}, \mathrm{S}$. et al. Phase diagram and electronic indication of high-temperature superconductivity at $65-\mathrm{K}$ in single-layer FeSe films. Nat. Mater. 12, 605-610 (2013).

17. Tan, S. et al. Interface-induced superconductivity and strain-dependent spin density waves in $\mathrm{FeSe} / \mathrm{SrTiO}_{3}$ thin films. Nat. Mater. 12, 634-640 (2013).

18. Ge, J.-F. et al. Superconductivity above $100 \mathrm{~K}$ in single-layer FeSe films on doped $\mathrm{SrTiO}_{3}$. Nat. Mater. 14, 285-289 (2015).

19. Haule, K., Shim, J. H. \& Kotliar, G. Correlated electronic structure of $\mathrm{LaO}_{1-x} \mathrm{~F}_{x} \mathrm{FeAs}$ Phys. Rev. Lett. 100, 226402 (2008).

20. Haule, K. \& Kotliar, G. Coherence-incoherence crossover in the normal state of iron oxypnictides and importance of Hund's rule coupling. N. J. Phys. 11, 025021 (2009).

21. Yi, M. et al. Observation of temperature-induced crossover to an orbital-selective mott phase in $\mathrm{A}_{x} \mathrm{Fe}_{2-y} \mathrm{Se}_{2}(\mathrm{~A}=\mathrm{K}, \mathrm{Rb})$ superconductors. Phys. Rev. Lett. 110, 067003 (2013).

22. $\mathrm{Yi}, \mathrm{M}$. et al. Observation of universal strong orbital-dependent correlation effects in iron chalcogenides. Nat. Commun. 6, 7777 (2015).

23. Yin, Z. P., Haule, K. \& Kotliar, G. Kinetic frustration and the nature of the magnetic and paramagnetic states in iron pnictides and iron chalcogenides. Nat. Mater. 10, 932-935 (2011).

24. Fanfarillo, L. \& Bascones, E. Electronic correlations in Hund metals. Phys. Rev. B 92, 075136 (2015).

25. de'Medici, L., Giovannetti, G. \& Capone, M. Selective mott physics as a key to iron superconductors. Phys. Rev. Lett. 112, 177001 (2014).

26. Aichhorn, M., Biermann, S., Miyake, T., Georges, A. \& Imada, M. Theoretical evidence for strong correlations and incoherent metallic state in FeSe. Phys. Rev. $B$ 82, 064504 (2010).

27. Kostin, A. et al. Imaging orbital-selective quasiparticles in the Hund's metal state of FeSe. Nat. Mater. 17, 869-874 (2018).

28. Mandal, S., Zhang, P., Ismail-Beigi, S. \& Haule, K. How correlated is the FeSe/SrTiO system? Phys. Rev. Lett. 119, 067004 (2017).

29. Cao, H.-Y., Tan, S., Xiang, H., Feng, D. L. \& Gong, X.-G. Interfacial effects on the spin density wave in $\mathrm{FeSe} / \mathrm{SrTiO}_{3}$ thin films. Phys. Rev. B 89, 014501 (2014).

30. Park, H., Haule, K. \& Kotliar, G. Magnetic excitation spectra in $\mathrm{BaFe}_{2} \mathrm{As}_{2}$ : a twoparticle approach within a combination of the density functional theory and the dynamical mean-field theory method. Phys. Rev. Lett. 107, 137007 (2011). 
31. Zhang, C. et al. Effect of pnictogen height on spin waves in iron pnictides. Phys. Rev. Lett. 112, 217202 (2014).

32. Moon, C.-Y., Park, H., Haule, K. \& Shim, J. H. Origin of doping-induced suppression and reemergence of magnetism in LaFeAsO ${ }_{1-x} \mathrm{H}_{x}$. Phys. Rev. B 94, 224511 (2016).

33. Liu, K., Lu, Z.-Y. \& Xiang, T. Atomic and electronic structures of FeSe monolayer and bilayer thin films on $\mathrm{SrTiO}_{3}$ (001): first-principles study. Phys. Rev. B 85, 235123 (2012).

34. Cao, H.-Y., Chen, S., Xiang, H. \& Gong, X.-G. Antiferromagnetic ground state with pair-checkerboard order in FeSe. Phys. Rev. B 91, 020504(R) (2015).

35. Zhou, Y. et al. Antiferromagnetic order in epitaxial FeSe films on $\mathrm{SrTiO}_{3}$. Phys. Rev. Lett. 120, 097001 (2018).

36. Liu, D. et al. Electronic origin of high-temperature superconductivity in singlelayer FeSe superconductor. Nat. Commun. 3, 931 (2012)

37. Nekrasov, I. A., Pavlov, N. S., Sadovskii, M. V. \& Slobodchikov, A. A. Electronic structure of FeSe monolayer superconductors. Low Temp. Phys. 42, 891-899 (2016).

38. Nekrasov, I. A., Pavlov, N. S. \& Sadovskii, M. V. On the origin of the shallow and 'replica' bands in FeSe monolayer superconductors. Jetp Lett. 105, 370-374 (2017).

39. Chen, X., Maiti, S., Linscheid, A. \& Hirschfeld, P. J. Electron pairing in the presence of incipient bands in iron-based superconductors. Phys. Rev. B 92, 224514 (2015).

40. Linscheid, A., Maiti, S., Wang, Y., Johnston, S. \& Hirschfeld, P. J. High $T_{c}$ via spin fluctuations from incipient bands: application to monolayers and intercalates of FeSe. Phys. Rev. Lett. 117, 077003 (2016).

41. Huang, D. \& Hoffman, J. E. Monolayer FeSe on $\mathrm{SrTiO}_{3}$. Annu. Rev. Condens. Matter. Phys. 8, 311-336 (2017)

42. Kuroki, K. et al. Unconventional pairing originating from the disconnected fermi surfaces of superconducting $\mathrm{LaFeAsO}_{1-x} \mathrm{~F}_{x}$. Phys. Rev. Lett. 101, 087004 (2008).

43. Maier, T. A., Graser, S., Hirschfeld, P. J. \& Scalapino, D. J. $d$-wave pairing from spin fluctuations in the $\mathrm{K}_{x} \mathrm{Fe}_{2-y} \mathrm{Se}_{2}$ superconductors. Phys. Rev. B 83, 100515(R) (2011).

44. Wang, F. et al. The electron pairing of $\mathrm{K}_{x} \mathrm{Fe}_{2-y} \mathrm{Se}_{2}$. Europhys. Lett. 93, 57003 (2011).

45. Saito, T., Onari, S. \& Kontani, H. Emergence of fully gapped $s_{++}$-wave and nodal $d$ -wave states mediated by orbital and spin fluctuations in a ten-orbital model of $\mathrm{KFe}_{2} \mathrm{Se}_{2}$. Phys. Rev. B 83, 140512(R) (2011).

46. Kang, J. \& Fernandes, R. M. Superconductivity in FeSe thin films driven by the interplay between nematic fluctuations and spin-orbit coupling. Phys. Rev. Lett. 117, 217003 (2016).

47. Mazin, I. I. Symmetry analysis of possible superconducting states in $\mathrm{K}_{x} \mathrm{Fe}_{y} \mathrm{Se}_{2}$ superconductors. Phys. Rev. B 84, 024529 (2011).

48. Khodas, M. \& Chubukov, A. V. Interpocket pairing and gap symmetry in Fe-based superconductors with only electron pockets. Phys. Rev. Lett. 108, 247003 (2012).

49. Fanfarillo, L., Giovannetti, G., Capone, M. \& Bascones, E. Nematicity at the Hund's metal crossover in iron superconductors. Phys. Rev. B 95, 144511 (2017).

50. Haule, K., Yee, C.-H. \& Kim, K. Dynamical mean-field theory within the fullpotential methods: electronic structure of Celrln ${ }_{5}, C$ Coln ${ }_{5}$, and CeRhln ${ }_{5}$. Phys. Rev. B 81, 195107 (2010).

51. Blaha, P., Schwarz, K., Madsen, G., Kvasnicka, D. \& Luitz, J. WIEN2k (Vienna University of Technology, Vienna, 2001).

52. Ceperley, D. M. \& Alder, B. J. Ground state of the electron gas by a stochastic method. Phys. Rev. Lett. 45, 566 (1980).

53. Perdew, J. P. \& Wang, Y. Accurate and simple analytic representation of the electron-gas correlation energy. Phys. Rev. B 45, 13244 (1992).

54. Haule, K. Quantum Monte Carlo impurity solver for cluster dynamical mean-field theory and electronic structure calculations with adjustable cluster base. Phys. Rev. B 75, 155113 (2007)
55. Baldini, E. et al. Discovery of the soft electronic modes of the trimeron order in magnetite. Nat. Phys. 16, 541-545 (2020).

56. Nomura, T. et al. Crystallographic phase transition and high- $\mathrm{T}_{c}$ superconductivity in LaFeAsO:F. Supercond. Sic. Technol. 21, 125028 (2008).

57. Haule, K. \& Pascut, G. L. Forces for structural optimizations in correlated materials within a DFT+embedded DMFT functional approach. Phys. Rev. B 94, 195146 (2016).

\section{ACKNOWLEDGEMENTS}

This research was supported by the Basic Science Research Program through the National Research Foundation of Korea (NRF) funded by the Ministry of Science and ICT (2016R1C1B1014715).

\section{AUTHOR CONTRIBUTIONS}

C.-Y.M. conceived the project, performed calculations, analyzed data, and wrote the papaer.

\section{COMPETING INTERESTS}

The author declares no competing interests.

\section{ADDITIONAL INFORMATION}

Supplementary information is available for this paper at https://doi.org/10.1038/ s41524-020-00414-3.

Correspondence and requests for materials should be addressed to C.-Y.M.

Reprints and permission information is available at http://www.nature.com/ reprints

Publisher's note Springer Nature remains neutral with regard to jurisdictional claims in published maps and institutional affiliations.

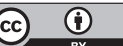

Open Access This article is licensed under a Creative Commons Attribution 4.0 International License, which permits use, sharing, adaptation, distribution and reproduction in any medium or format, as long as you give appropriate credit to the original author(s) and the source, provide a link to the Creative Commons license, and indicate if changes were made. The images or other third party material in this article are included in the article's Creative Commons license, unless indicated otherwise in a credit line to the material. If material is not included in the article's Creative Commons license and your intended use is not permitted by statutory regulation or exceeds the permitted use, you will need to obtain permission directly from the copyright holder. To view a copy of this license, visit http://creativecommons. org/licenses/by/4.0/.

(c) The Author(s) 2020 